\title{
A compendium of transformation formulas useful in GPS work
}

\author{
T. Soler \\ National Geodetic Survey, NOS, NOAA, N/NGS22, \#8116, 1315 East-West Highway, Silver Spring, MD 20910-3282, USA \\ Tel: +1 3017133205 ext. 157; fax: +1 301713 4322; e-mail: tom@ngs.noaa.gov \\ Received: 16 June 1997 / Accepted: 22 April 1998
}

\begin{abstract}
In order properly to apply transformations when using data derived from different GPS solutions, the effects of plate motion on the coordinates should be accurately taken into consideration. Only then can a rigorous comparison be established between results observed at different epochs. Equations are given to relate GPS-derived Cartesian coordinates and velocities affected by changes of their reference frames and epochs.
\end{abstract}

Key words. Transformation of frames - Effect of plate tectonics on GPS observables - Active and passive transformations $\cdot$ Station velocities

\section{Introduction}

Accurate positioning using modern Global Positioning System (GPS) technology and methods requires the full understanding of reference-frame transformations. The fact that GPS terrestrial observing stations are located on moving lithospheric plates slightly complicates the issue. Recent developments in space geodesy techniques have reached a level of sophistication capable of corroborating to a high degree of certainty the geophysical theories describing global plate tectonics. Absolute motions of points on the Earth's surface with respect to a fixed geocentric coordinate frame average about $3 \mathrm{~cm} /$ year, and depend on geographic location. Crustal motions along subduction zones could be as large as $24 \mathrm{~cm} /$ year, the fastest crustal motions yet observed (Bevis et al. 1995). In addition, crustal deformations and earthquake activity near transform faults increase the difficulty of properly modeling crustal velocity fields (see, e.g., Snay et al. 1996). GPS data analysts are also aware that the original Cartesian vector components resulting from any GPS data reduction technique always refer to the satellite ephemeris terres- trial frame used during processing the GPS vectors. These vectors refer to a specific epoch $t_{0}$ and rigorous transformations are required when mixing data collected at different epochs intended to calculate geodetic positions.

The following conventions will be retained throughout this article:

1. Only right-handed, three-dimensional coordinate frames are used.

2. Geodetic longitude $\lambda$ is counted positive toward the east.

3. Positive rotations about axes (the three of a coordinate frame, or any arbitrary axis going through the origin of the frame) are anticlockwise (counterclockwise) rotations as viewed looking towards the origin of the coordinate frame.

With these definitions, rotations about coordinate axes are conveniently expressed by proper orthogonal matrices denoted $R_{i}(\theta), i=1,2,3$ (e.g., Kaula 1966, p. 13). The subscripts indicate rotations about the first, second, and third axis, respectively. The argument represents the magnitude of the rotation, which can be any finite angle $\theta$. Successive rotations are operated in sequence, however the final result is not commutative and depends on the specific sequence of the individual rotations applied. Exceptions to this rule are differential rotations, which follow the commutative property.

Rotations of geocentric vectors about an arbitrary axis - while keeping the geocentric coordinate frame fixed - are required properly to account for plate tectonic motions. Every differential displacement caused by a small rotation could be associated with a skew-symmetric (antisymmetric) matrix which is a function of the angular rotation components. According to the foregoing definitions, and in order to simplify as much as possible the nomenclature, the following conventions are introduced. When anticlockwise rotation of frames is involved, the three differential rotations about the three Cartesian axes $x, y$, and $z$, will be denoted, respectively, by $\epsilon_{x}, \epsilon_{y}$, and $\epsilon_{z}$. However, when rotations of geocentric 
vectors (i.e., points) around arbitrary axes are performed - keeping the geocentric frame fixed - these rotations are expressed by the components (referred to the fixed Cartesian frame) of the angular velocity vector, namely, $\Omega_{x}, \Omega_{y}$, and $\Omega_{z}$.

A compact notation for $3 \times 3$ skew-symmetric matrices will be used throughout. When a vector is differentially rotated about any axis with respect to a fixed frame - active point of view or active transformation - its components will be operated through the following skew-symmetric matrix:

$[\Omega]=\left[\begin{array}{ccc}0 & -\Omega_{z} & \Omega_{y} \\ \Omega_{z} & 0 & -\Omega_{x} \\ -\Omega_{y} & \Omega_{x} & 0\end{array}\right]$

The foregoing notation is consistent with the definition of fixed right-handed coordinate systems and positive anticlockwise rotation of vectors, which is labeled by some authors as the right-hand rule. This sign convention is the one primarily used in rigid body mechanics and widely adopted by geophysicists investigating plate kinematics. It should be kept in mind that when one rotates geocentric vectors using the matrix in Eq. (1), the Cartesian axes remain fixed, although the components of the vector will change because its tip is moved to a new position (see Fig. 1).

The opposite scenario occurs when coordinate axes are differentially rotated keeping the vector (or the location of the point) fixed in space - passive transformation - then the physical position of the vector remains invariant in space, although its components change due to the rotation of the coordinate axes (see Fig. 2). It can be proved that anticlockwise rotation of axes is equiv-

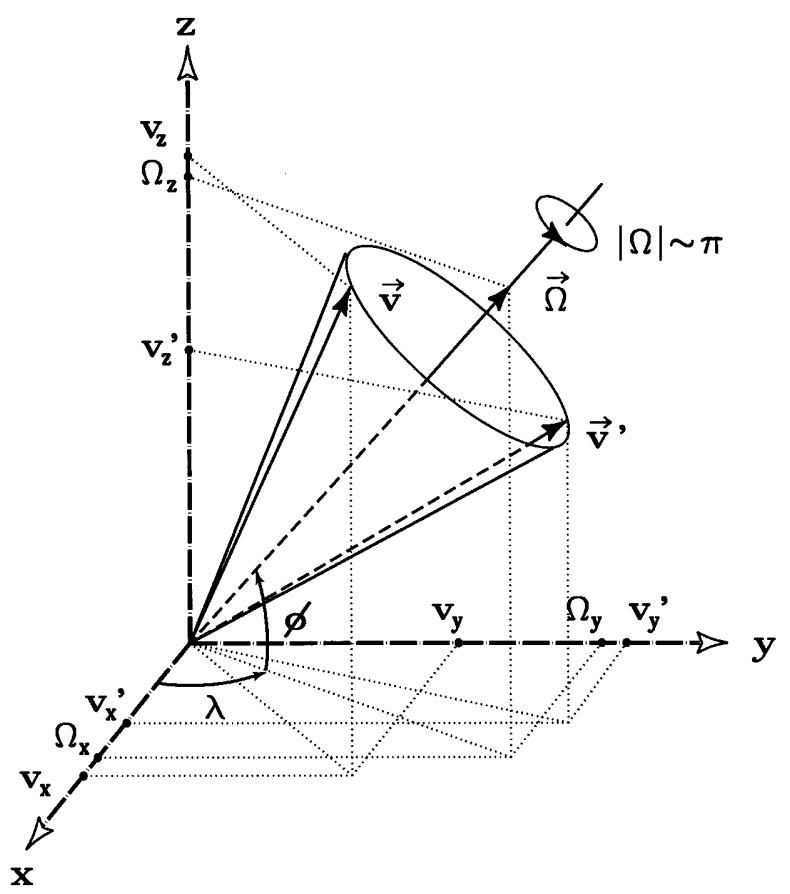

Fig. 1. Active transformation of vectors (frame remains fixed; vector rotates)

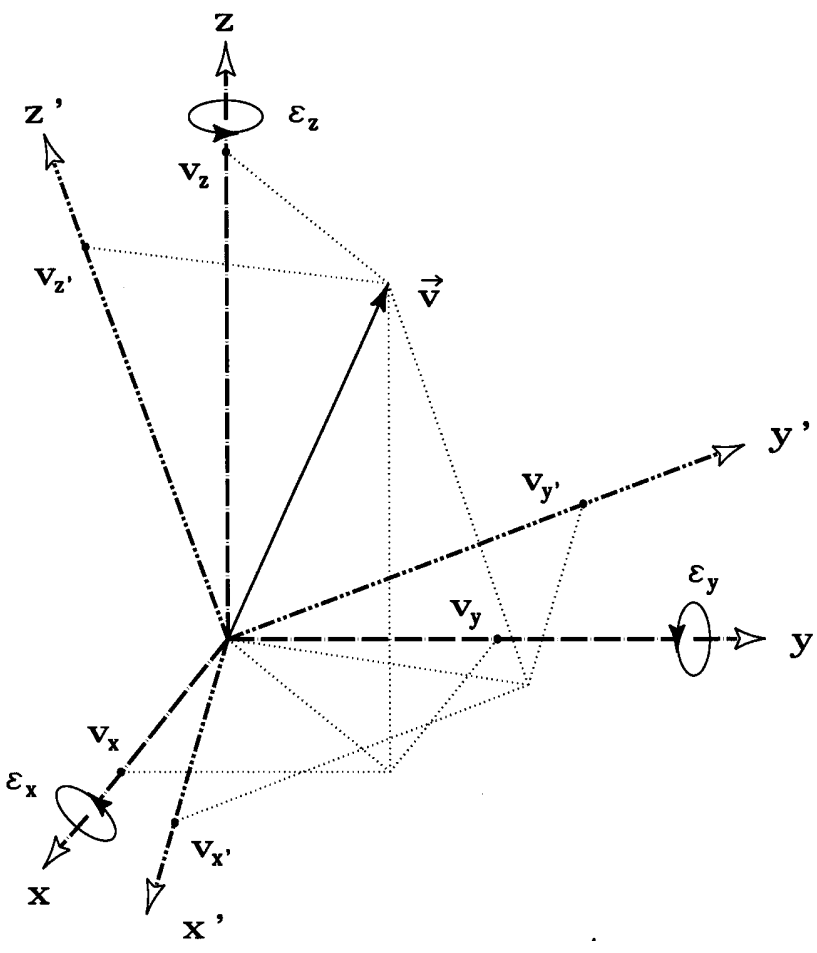

Fig. 2. Passive transformation of vectors (frame rotates; vector remains fixed)

alent to clockwise rotation of vectors and vice versa. However, since by definition we want to enforce the property that all positive rotations in three-dimensional space have the anticlockwise sense as positive, the rotation of coordinate frames by $\epsilon_{x}, \epsilon_{y}$, and $\epsilon_{z}$ should have a skew-symmetric matrix with opposite sign to the one shown in Eq. (1), i.e.,

$[\epsilon]^{t}=\left[\begin{array}{ccc}0 & \epsilon_{z} & -\epsilon_{y} \\ -\epsilon_{z} & 0 & \epsilon_{x} \\ \epsilon_{y} & -\epsilon_{x} & 0\end{array}\right]$

Through basic matrix algebra it is known that $[\epsilon]^{t}=-[\epsilon]$, where $t$ denotes matrix transpose. The matrices of Eqs. (1) and (2) are, respectively, consistent with positive anticlockwise rotation of vectors around a single arbitrary axis (frame fixed) rotating with angular velocity $\Omega$ or, equivalently, anticlockwise rotations (vector fixed) $\epsilon_{x}, \epsilon_{y}, \epsilon_{z}$ about three-dimensional coordinate axes.

Because this discussion is restricted to three-dimensional Euclidean space, $3 \times 1$ three-dimensional column (vector) matrices will be abbreviated as follows: $\{x\}=\left\{\begin{array}{lll}x & y z\end{array}\right\}^{t},\left\{T_{x}\right\}=\left\{T_{x} T_{y} T_{z}\right\}^{t}$, etc. Notice that the ordered sequence of coordinates is retained, although only the first coordinate appears explicitly in the abbreviated notation. This direct notation is a short, compact way of representing vector matrices, and has the advantage of maintaining the matrix nomenclature popular among aerospace and astronautical engineering textbooks. The $3 \times 3$ identity (unit) matrix is denoted by [I] and the $3 \times 3$ zero (null) matrix by [0]. 


\section{Geocentric terrestrial (Earth-fixed) conventional reference frames}

With the advent of GPS the possibility of accurately (to the subcentimeter level) determining three-dimensional Cartesian coordinates has drastically changed the methodology used in geodesy, surveying, and mapping applications. However, the use of an adopted "best" mean-Earth fitting ellipsoid of revolution (e.g., WGS84, GRS80) will be required in order to express results in curvilinear geodetic coordinates $(\lambda, \phi, h)$. The need for geodetic longitude, geodetic latitude, and geodetic (ellipsoid) height is still useful because they are more intuitive, facilitating the graphic depiction of points on the surface of the Earth through mapping. However, although more abstract in concept, three-dimensional coordinates are the primary output of any GPS reduction process and the user should be familiar with how they are employed and, more importantly, how they are rigorously transformed from frame to frame.

The conversion from curvilinear geodetic $(\lambda, \phi, h)$ to Cartesian $(x, y, z)$ coordinates is given by the well-known equations:

$$
\left\{\begin{array}{l}
x \\
y \\
z
\end{array}\right\}=\left\{\begin{array}{c}
(N+h) \cos \phi \cos \lambda \\
(N+h) \cos \phi \sin \lambda \\
{\left[N\left(1-e^{2}\right)+h\right] \sin \phi}
\end{array}\right\}
$$

where $N$ is the principal radius of curvature along the prime vertical (see Sect. 7). The inverse transformation (Cartesian to geodetic) does not have a simple explicit closed formulation. A computer subroutine based on the approached suggested by Borkowsky (1989) is given in (McCarthy 1996, p. 12).

At the time of writing there are two immediate choices of geocentric (Cartesian) conventional terrestrial reference frames: WGS84 (G873) and ITRF96. WGS84 (G873), epoch 1997.0, is the latest realization of a series of WGS84 frames implemented by the National Imagery and Mapping Agency (NIMA, formerly Defense Map- ping Agency). The letter G stands for "GPS," implying that archived Doppler data were excluded from the observations materializing the frame; the " 873 " indicates the GPS week number $\left(0^{h}\right.$ UTC, 29 September 1996) of the initial date when the coordinate frame was made available through NIMA GPS precise ephemerides. The same frame was incorporated into the Keplerian elements of the broadcast message on 29 January 1997 (Malys et al. 1997a).

The other alternative is the International Earth Rotation Service (IERS) Terrestrial Reference Frame ITRF96, epoch 1997.0 (available since $0^{h}$ UTC, 1 March 1998). This solution includes extraterrestrial data from several sources (VLBI, SLR, GPS, and DORIS) collected up to year 1997. ITRF frames are created under international sponsorship and satisfy accuracy requirements for various modern space techniques (Feissel and Gambis 1993). Related to each ITRF frame there is an associated velocity field, i.e., each station of the network materializing the frame has a velocity vector $\left(v_{x}, v_{y}, v_{z}\right)$ indicating its time-dependent absolute displacement caused by the motion of the tectonic plate on which the point is located. These secular displacements can be approximated anywhere on the Earth's crust by spherical geophysical models such as NNR-NUVEL1A (DeMets et al. 1994) which is a recent revised improvement of the original NUVEL-1 (Argus and Gordon 1991).

\section{Local reference frames}

Several local Cartesian frames can be defined at any observation point $A$. Obviously, local terrestrial threedimensional frames parallel to WGS84 or ITRF96 at $A$ would be required to refer the vector components between the standpoint (reference or base station) $A$ and the forepoint (remote station) $B:\left\{x_{B}-x_{A} y_{B}-y_{A}\right.$ $\left.z_{B}-z_{A}\right\}^{t} \equiv\left\{x_{B}-x_{A}\right\}^{t}=\{x\}^{t}$ (see Fig. 3). These are the typical vector components used as GPS observables in

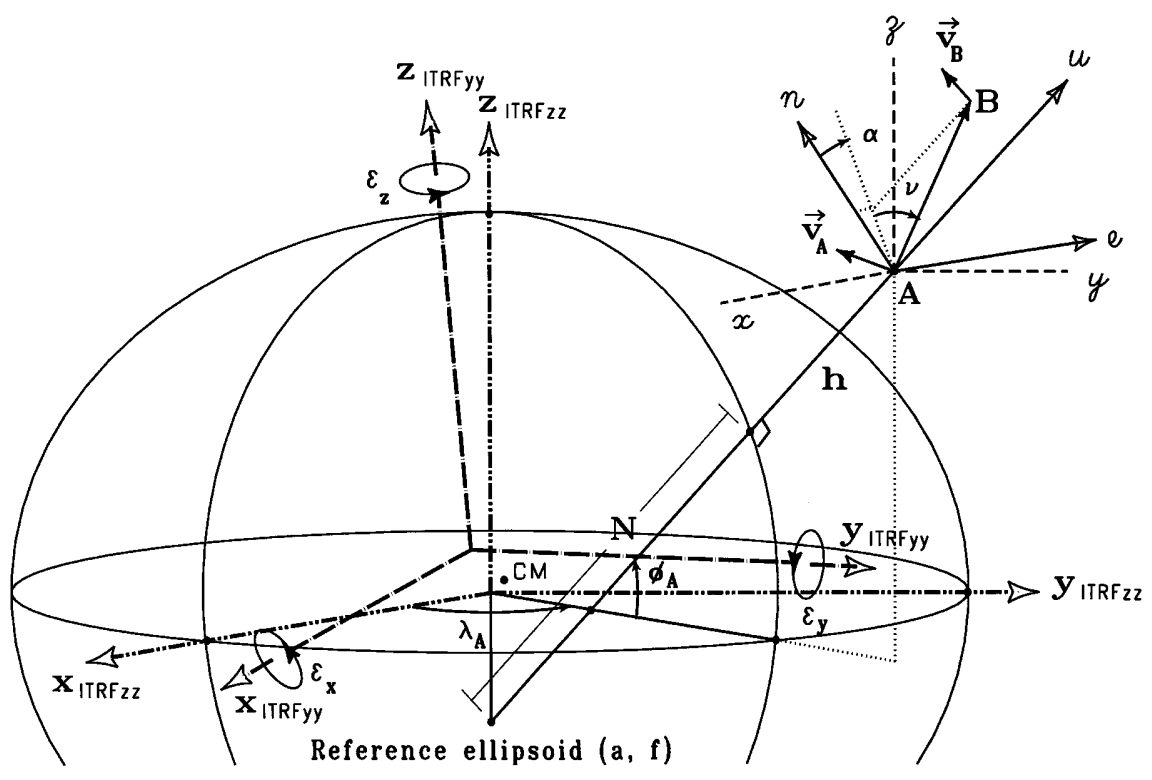

Fig. 3. Geocentric terrestrial, local terrestrial, and local geodetic frames 
three-dimensional network adjustments during the final stages of a GPS project.

Another convenient frame to use at any GPS observation point $A$ is the local geodetic frame $(e, n, u)$ shown in Fig. 3, defined as follows:

1. origin: any point $A(x, y, z) \equiv A(\lambda, \phi, h)$, referred to a given ellipsoid;

2. $u$-axis: normal through $A$ to the reference ellipsoid; the positive sign is in the outward (geodetic zenith) or "up" direction;

3. $e$-axis: normal to $u$ and the geodetic meridian plane (when $h=0$, tangent to the geodetic parallel through $A$ ); positive (east) is in the direction of increasing $\lambda$;

4. $n$-axis: perpendicular to $e$ and $u$ forming a righthanded triad (when $h=0$, tangent to the geodetic meridian through $A$ ). Positive (north) is in the direction of increasing $\phi$; the plane $e-n$ defines the local geodetic horizon.

This local geodetic frame is commonly alluded to in practical applications as the east-north-up frame (righthanded convention enforced). Some authors use the terminology "vertical" instead of "up," which is satisfactory if one is fully aware that the measurements are purely geometric and are given along the normal to the ellipsoid with no relation to the curved plumb line.

\section{Transformation between local geocentric terrestrial and local geodetic frames}

Assuming that an adopted ellipsoid of revolution (e.g., WGS84, GRS80) is centered at the origin of the geocentric conventional terrestrial reference frame, the transformation of components of a vector $\overrightarrow{A B}$ between local terrestrial ( $x y z)$ and local geodetic (e $n u)$ frames could be obtained as follows:

$$
\{e\}=[R]\left\{x_{B}-x_{A}\right\}=[R]\{x\}
$$

where the rotation matrix $[R]$ of the transformation is given by

$$
\begin{aligned}
{[R] } & =R_{1}\left(\frac{1}{2} \pi-\phi_{A}\right) R_{3}\left(\lambda_{A}+\frac{1}{2} \pi\right) \\
& =R_{3}\left(\frac{1}{2} \pi\right) R_{2}\left(\frac{1}{2} \pi-\phi_{A}\right) R_{3}\left(\lambda_{A}\right)
\end{aligned}
$$

or explicitly

$[R]=\left[\begin{array}{ccc}-\sin \lambda & \cos \lambda & 0 \\ -\sin \phi \cos \lambda & -\sin \phi \sin \lambda & \cos \phi \\ \cos \phi \cos \lambda & \cos \phi \sin \lambda & \sin \phi\end{array}\right]_{A}$

Related to the local geodetic frame are the following two geometric parameters:

1. $\alpha$ : geodetic azimuth; angle in the plane of the local geodetic horizon counted positive clockwise from geodetic north; $0 \leq \alpha \leq 2 \pi$;

2. $v$ : vertical angle; angle in the plane containing the geodetic vertical (i.e., normal to the ellipsoid) and the forepoint measured from the local geodetic horizon to the direction between the two points and counted positive above the geodetic horizon; $-\frac{1}{2} \pi \leq v \leq \frac{1}{2} \pi$; the geodetic zenith distance $z$ is defined as $z=\frac{1}{2} \pi-v$, $0 \leq z \leq \pi$.

These two parameters can be computed very accurately using GPS from the values $\{e\}$ in Eq. (4) by the wellknown formulas:

$\tan \alpha=e / n$

$\tan v=u /\left(e^{2}+n^{2}\right)^{\frac{1}{2}}$

Physical quantities such as astronomic azimuth $\alpha^{*}$ and astronomic altitude $v^{*}$ are related to $\alpha$ and $v$ through the components of the deflection of the vertical $(\eta, \xi)$ (Heiskanen and Moritz 1967, p. 186 and 190).

$\alpha^{*}=\alpha+\eta \tan \phi+(\xi \sin \alpha-\eta \cos \alpha) \tan v$

$v^{*}=v+\xi \cos \alpha+\eta \sin \alpha$

Thanks to the great accuracy achieved by GPS, the required components $\eta$ and $\xi$ could be determined completely independent of classical astronomic and/or gravimetric methods using GPS and leveling (Soler et al. 1989). Incidentally, the so-called Laplace equation (or condition) is merely $\Delta \alpha=\alpha^{*}-\alpha$.

An important formula relates $h$ (measured through GPS) with $H$ (orthometric height, determined through observations of leveling and gravity) and $N_{g}$ (predicted undulation or geoid height):

$h \approx H+N_{g}$

\section{Transformations between geocentric conventional terrestrial frames}

When transforming between geocentric conventional terrestrial frames, one should also consider that these frames are given at some specific epoch. As already explained, this precaution is required to take into account the motion of the observing stations which is inevitable due to the phenomena of plate tectonics. Points on the ITRFyy, epoch $t_{0}$ ( $y y$ denotes the last two digits of the ITRF yearly solution, e.g., ITRF96) series of coordinate frames have attached a velocity field giving at every point the corresponding components of the linear velocity about the three local terrestrial Cartesian axes: $\left(v_{x}, v_{y}, v_{z}\right)$.

Many of the ITRF stations belong to the International GPS Service for Geodynamics (IGS) network which tracks the satellites of the GPS constellation (Neilan 1997). The location of these receivers, as well as, e.g. the National Geodetic Survey (NGS) Continuously Operating Reference Stations (CORS) network (Strange 1995), are useful as "fiducial" stations to propagate coordinates in GPS surveys. The term "fiducial" is loosely applied to describe continuously operating GPS sites whose RINEX2 data are made available 
electronically to the geodetic-surveying community. Generally, the coordinates and velocities of these permanent sites are accurately known with respect to some predefined reference frame and could be used to rigorously propagate coordinates to other arbitrary points.

The rigorous transformation between point coordinates from ITRFyy, epoch $t_{0}$, to ITRFzz, epoch $t$, (designated symbolically by the mapping: $\left.\operatorname{ITRF} y y\left(t_{0}\right) \rightarrow \operatorname{ITRF} z z(t)\right)$ could be implemented according to the equation (see Appendix):

$$
\begin{aligned}
\{x\}_{\text {ITRF } z z}= & \left\{T_{x}\right\}+(1+s) \llbracket[\epsilon]^{t}+[I] \rrbracket \\
& \times \llbracket\{x\}_{\text {ITRF } y y}+\left\{v_{x}\right\}_{\text {ITRF } y y}\left(t-t_{0}\right) \rrbracket
\end{aligned}
$$

where: (1) $\left\{T_{x}\right\}=$ coordinates of the origin of the frame ITRF $y y$ in the frame ITRF $z z$, i.e., the translations or shifts between the two frames, (2) $\epsilon_{x}, \epsilon_{y}, \epsilon_{z}=$ differential anticlockwise rotations (expressed in radians), respectively, around the axes $x, y$, and $z$ of the ITRF $y y$ frame to establish parallelism with the ITRF $z z$ frame, and (3) $s=$ differential scale change (expressed in $\mathrm{ppm} \times 10^{-6}$; ppm $=$ parts per million). The Cartesian coordinates $\{x\}$ and the velocities $\left\{v_{x}\right\}$ must have conformable units. The interval of time $t-t_{0}$ is generally expressed in year and its fraction. Note that $t$ could be the actual time of the GPS observations (e.g., $t=1998.3587$ ).

Furthermore, if ITRFyy=ITRF93, the following matrix expression (see Appendix) should be added to Eq. (12):

$$
\begin{aligned}
& \llbracket\left\{\dot{T}_{x}\right\}+\llbracket(1+s)[\dot{\epsilon}]^{t}+\dot{s}\left[[\epsilon]^{t}+[I]\right] \rrbracket\{x\}_{\text {ITRF93 }} \rrbracket \\
& \quad \times\left(t-t_{0}\right)
\end{aligned}
$$

Included in the preceding are the rates of the ITRF93 translation, rotation, and scale parameters required for $y y=93$ because of the decision of IERS to make consistent the time evolution of the ITRF93 frame with the IERS series of Earth orientation parameters (EOP). The seven transformation parameters required in Eqs. (12) and (13) are tabulated in Boucher and Altamimi (1996). A note of caution is in order; all rotations involved in the equations described herein are consistent with anticlockwise positive rotations, while, contrary to standard practice, clockwise positive rotations are implied in the values given by Boucher and Altamimi (1996). On this issue read the exchange of letters "to the editor" in GPS World (1997).

Similarly, from Eq. (A5) in the Appendix the rigorous transformation of velocities can be expressed as:

$$
\begin{aligned}
\left\{v_{x}\right\}_{\mathrm{ITRF} z z}= & \llbracket(1+s)[\dot{\epsilon}]^{t}+\dot{s}\left[[\epsilon]^{t}+[I]\right] \rrbracket\{x\}_{\text {ITRF } y y} \\
& +(1+s) \llbracket[\epsilon]^{t}+[I] \rrbracket\left\{v_{x}\right\}_{\mathrm{ITRF} y y}
\end{aligned}
$$

Sometimes, the values of $\left\{v_{x}\right\}$ are not readily known. This is the situation for GPS stations which are not part of the set of CORS or ITRF global sites. Then, approximations could be obtained by using any of the published kinematic plate models. In such case, the angular velocity components $\left\{\Omega_{x}\right\}_{P_{i}}$ for each plate $P_{i}$, are known quantities that could be extracted from the available geophysical models. Then, the approximation for the velocity vector $\left\{v_{x}\right\}$ required in Eqs. (12) and (14) is determined as follows

$$
\left\{v_{x}\right\}_{\mathrm{ITRF} y y} \approx[\Omega]_{P_{i}}\{x\}_{\mathrm{ITRF} y}
$$

The elements of $[\Omega]_{P_{i}}$ are given in radians/year and contain angular velocity components of the particular plate $P_{i}$ on which the point is located. The components of $\left\{\Omega_{x}\right\}$ in $\mathrm{rad} / \mathrm{My}$ are tabulated in, e.g., McCarthy (1996, p. 14) for the model (no net rotation) NNRNUVEL1A. At a minimum, station velocities should be applied to the fiducial sites before starting GPS processing in order to bring the position of these reference stations as close as possible to their actual spatial location at the time the observations were collected. For consistency, the selected reference frame for all fiducial points should be the one implicit in the precise ephemeris used during processing, consequently the resulting coordinates are referred to the reference frame of the satellite orbits used and the actual epoch of observation. The final processed GPS results could be transformed to any other conventional terrestrial frame using Eq. (12).

If preferred, for better practical visualization, the velocity components could be expressed along the local east-north-up frame applying Eq. (4):

$\left\{v_{e}\right\}=[R]\left\{v_{x}\right\}$

\section{Anticlockwise rotation of geocentric vectors around geocentric arbitrary axes}

Assume that one wants to rotate a geocentric vector of components $\left\{\mathbf{v}_{x}\right\}$ (in other words, a point $P$ of coordinates $\{x\}$ ), by an anticlockwise rotation of magnitude $\Omega$ (given in radians) around an axis with direction cosines $\{\ell\}$. Direction cosines are used to define the orientation of a line in space. Each spatial line going through the origin of the geocentric Cartesian frame forms three well-defined angles with the Cartesian axes. The cosine of each one of these angles is termed direction cosine. They will be denoted in matrix notation by $\{\ell\}=\left\{\ell_{1} \ell_{2} \ell_{3}\right\}^{t}$. Recall that the direction cosines of a line through a point with geodetic coordinates $P(\lambda, \phi, h)$ are given by: $\ell_{1}=\cos \lambda \cos \phi, \quad \ell_{2}=\sin \lambda \cos \phi$, and $\ell_{3}=\sin \phi$. They are the components of the unit vector along the normal to the ellipsoid going through $P$.

The formulation for active transformations was originally introduced by Euler (1775) and revived a century later by Thomson and Tait (1879). The rotation matrix of the transformation takes the form:

$R_{\ell}(\Omega)=\cos \Omega[I]+\sin \Omega[\ell]+(1-\cos \Omega)[\ell]^{2}$

where $[\ell]$ is given according to the definitions of direction cosines $\ell_{1} \equiv \ell_{x}, \ell_{2} \equiv \ell_{y}, \ell_{3} \equiv \ell_{z}$ and Eq. (2).

When anticlockwise rotation of Cartesian axes of frames (passive transformation) is dictated, the transpose rotation matrix $R_{\ell}^{t}(\Omega)$, not $R_{\ell}(\Omega)$, should be used. Applying to $R_{\ell}^{t}(\Omega)$ the particular case of the direction 
cosines of the three Cartesian axes (i.e., $\ell_{1}=1, \ell_{2}=0$, $\ell_{3}=0$, for the $x$-axis, etc.), the three standard rotation matrices $R_{i}(\theta), i=1,2,3$, about the reference axes described in the Introduction are obtained. Notice that in Eq. (17) no assumptions about the magnitude of $\Omega$ were made, $0 \leq \Omega \leq 2 \pi$.

Reviewing:

1. Active transformation (body-axes rotation). Coordinate (vector component) transformation with the Cartesian axes fixed in space and the point carried with the body (see Fig. 1):

$$
\left\{\mathbf{v}_{x}\right\} \rightarrow\left\{\mathbf{v}_{x}^{\prime}\right\} \equiv\left\{\mathbf{v}_{x}^{\prime}\right\}=R_{\ell}(\Omega)\left\{\mathbf{v}_{x}\right\}
$$

2. Passive transformation (space-axes rotation). Coordinate (vector component) transformation with the point (body) fixed in space and the Cartesian axes rotating (see Fig. 2):

$$
\left\{\mathbf{v}_{x}\right\} \rightarrow\left\{\mathbf{v}_{x^{\prime}}\right\} \equiv\left\{\mathbf{v}_{x^{\prime}}\right\}=R_{\ell}^{t}(\Omega)\left\{\mathbf{v}_{x}\right\}
$$

The change $\{d x\}$ in the Cartesian coordinates of a point $\{x\}$ due to a rotation $\Omega$ about an arbitrary axis of direction cosines $\{\ell\}$ given in the sense (new-old) could be obtained through the equation:

$$
\{d x\}=\left[R_{\ell}(\Omega)-[I]\right]\{x\}
$$

If the angular rotation $\Omega$ is small $(\sin \Omega \approx \Omega ; \cos \Omega \approx 1)$, the preceding expression reduces to

$$
\{\delta x\}=[\Omega]\{x\}
$$

which also applies to the determination of velocity components as described above (Eq. 15) when the units of $\Omega$ are, e.g. radians/year.

The transformation from a general rotation matrix $[\Re]$ with direction cosines $\ell_{i j}$ to a rotation $R_{\ell}(\Omega)$ around an axis with direction cosines $\ell_{i}, i=1,2,3$, and angle $\Omega$ is given according to the equations:

$\cos \Omega=\frac{1}{2}[\operatorname{tr}[\Re]-1]$

where $\operatorname{tr}[\mathfrak{R}]$ denotes the trace of $[\mathfrak{R}]$, the sum of the elements of its diagonal, e.g. $\operatorname{tr}[\mathfrak{R}]=\Sigma \ell_{i i}$, and

$\ell_{1}=\frac{1}{2}\left(\ell_{23}-\ell_{32}\right) / \sin \Omega$

$\ell_{2}=\frac{1}{2}\left(\ell_{31}-\ell_{13}\right) / \sin \Omega$

$\ell_{3}=\frac{1}{2}\left(\ell_{12}-\ell_{21}\right) / \sin \Omega$

where clearly the case $\sin \Omega=0$ is excluded.

An equation equivalent to Eq. (17) could also be obtained by simple application of successive rotations about axes, keeping in mind the difference between passive and active transformations. Notice that a rotation of magnitude $\Omega$ about an arbitrary axis as depicted in Fig. 1 can be derived using the following steps:
1. passive anticlockwise rotation about the $z$-axis by $\lambda: R_{3}(\lambda)$

2. passive clockwise rotation about the new rotated $y$ axis by $\phi: R_{2}(-\phi)=R_{2}^{t}(\phi)$;

3. active anticlockwise rotation about the new rotated $x$ axis by $\Omega: R_{1}^{t}(\Omega)=R_{1}(-\Omega)$.

Undo steps (2) and (1), that is:

4. passive anticlockwise rotation about the rotated $y$-axis by $\phi: R_{2}(\phi)$;

5. passive clockwise rotation about the rotated $z$-axis by $\lambda: R_{3}(-\lambda)=R_{3}^{t}(\lambda)$.

Thus, once all individual rotations are taken into consideration in a sequential manner, the resulting proper orthogonal (rotation matrix) operator could be written:

$R_{\ell}(\Omega)=R_{3}(-\lambda) R_{2}(\phi) R_{1}(-\Omega) R_{2}(-\phi) R_{3}(\lambda)$

It can be easily proved that this equation is identical to Eq. (17) once the substitutions $\ell_{x}=\cos \lambda \cos \phi$; $\ell_{y}=\sin \lambda \cos \phi ; \ell_{z}=\sin \phi$ are implemented.

In general, the order and sense of the rotations described in Eq. (22) will depend on how the angles $\lambda$ and $\phi$ are defined. This alternative formalism to compute the rotation operator about an arbitrary axis was implicitly applied in Mueller (1969, p. 115) when describing the corrections due to stellar proper motion. The sign conventions used here for all rotations are consistent with the standard definitions followed throughout the book.

\section{Local displacements of a point in curvilinear coordinates due to plate rotations}

Sometimes one may be interested in knowing the changes in the curvilinear coordinates at any arbitrary point $P(\lambda, \phi, h) \equiv P(x, y, z)$ due to plate motions. The differential changes (per year) in longitude, latitude (both in radians), and ellipsoid height (in linear units), i.e., $\{\delta \lambda\}=\{\delta \lambda \delta \phi \delta h\}^{t}$ due to a differential rotation $\Omega$ $(\mathrm{rad} / \mathrm{year})$ can be computed as follows:

$$
\begin{aligned}
\{\delta \lambda\} & =[H]^{-1}[R][\Omega]_{P_{i}}\{x\}=[H]^{-1}[R]\left\{v_{x}\right\} \\
& =[H]^{-1}\left\{v_{e}\right\}
\end{aligned}
$$

where $[H]$ is the so-called Lamé's matrix and $[H]^{-1}$ denotes its inverse. For the particular case of orthogonal curvilinear (geodetic) coordinates treated here, the matrix $[H]$ is diagonal and given explicitly by (Soler 1976, p. 18):

$[H]=\left[\begin{array}{ccc}(N+h) \cos \phi & 0 & 0 \\ 0 & M+h & 0 \\ 0 & 0 & 1\end{array}\right]$

Notice that $[H]^{2}$ is equal to the "metric matrix" of tensor calculus. $N$ and $M$ are the principal radii of curvature along the prime vertical and meridian, respectively. They can be computed from the following exact equations: 


$$
\begin{aligned}
& N=a / W ; M=a\left(1-e^{2}\right) / W^{3} ; \\
& W=\left(1-e^{2} \sin ^{2} \phi\right)^{\frac{1}{2}} ; \text { and } e^{2}=2 f-f^{2},
\end{aligned}
$$

where $a$ and $f$ are the parameters describing the size (semi-major axis) and shape (flattening) of the adopted reference ellipsoid (e.g., for the WGS84 ellipsoid: $a=6378137 \mathrm{~m}$ and $1 / f=298.257223563$; the GRS80 ellipsoid has identical $a$ and $1 / f=298.257222101)$.

It can be proved that when Eq. (23) is simplified to the spherical case, i.e., $N+h \equiv M+h \equiv r$ (the approximate radius of the Earth's sphere), then $\delta r=0$, implying that, as expected, the displacements given by spherical plate rotation models only produce horizontal displacements on the surface of the sphere and changes along the radial (vertical) component are zero. That is not the case when plate rotations are applied to an ellipsoid of revolution.

\section{Transformation of observations}

Generally, the final post-processed products available to the GPS user are vector components $\left\{x_{B}-x_{A}\right\}_{t_{0}}$ at time $t_{0}$ between standpoint $A$ and forepoint $B$ referred to a local conventional terrestrial frame $\{x\}$, the one implicit in the satellite ephemeris employed. Applying the theory described above, it is possible to transform these components (the observables) to any other selected epoch $t$, previous to the implementation of a three-dimensional adjustment to determine coordinates for all points in the network. Since these GPS vector components are referred to a local frame, only rotations and scale affect the transformation, thus by analogy with Eq. (12):

$$
\begin{aligned}
\left\{x_{B}-x_{A}\right\}_{t}= & (1+s)\left[[\epsilon]^{t}+[I]\right] \llbracket\left\{x_{B}-x_{A}\right\}_{t_{0}} \\
& +\left\{v_{B_{x}}-v_{A_{x}}\right\}\left(t-t_{0}\right) \rrbracket
\end{aligned}
$$

where the values for the scale factor $s$ and the rotations $\epsilon_{x}, \epsilon_{y}, \epsilon_{z}$, correspond to the parameters implicit in the transformation between the conventional reference frames from epoch $t_{0}$ to $t$.

The final variance-covariance matrix of the new transformed GPS observations can be determined from the original values at $t_{0}$ according to the following equation:

$\Sigma_{\left\{x_{B}-x_{A}\right\}_{t}}=(1+s)^{2}\left[[\epsilon]^{t}+[I]\right] \llbracket \Sigma \rrbracket\left[[\epsilon]^{t}+[I]\right]^{t}$

where

$$
\begin{aligned}
\llbracket \Sigma \rrbracket= & \Sigma_{\left\{x_{B}-x_{A}\right\} t_{0}}+2\left(t-t_{0}\right) \\
& \times\left[\Sigma_{\left\{x_{B}-x_{A}\right\} t_{0} v_{B}}-\Sigma_{\left\{x_{B}-x_{A}\right\} t_{0} v_{A}}\right] \\
& +\left(t-t_{0}\right)^{2}\left[\Sigma_{v_{A}}+\Sigma_{v_{B}}-2 \Sigma_{v_{A} v_{B}}\right]
\end{aligned}
$$

These expressions are based on the application of the standard law of propagation of errors. In practical situations all elements in the variance-covariance matrix $\Sigma_{\left\{x_{B}-x_{A}\right\} t_{0}}$ are known. However, $\Sigma_{v_{A}}$ and $\Sigma_{v_{B}}$ are generally diagonal matrices and the values of the elements of the cross-correlation matrices are not available and could be neglected, i.e.,

$$
\Sigma_{\left\{x_{B}-x_{A}\right\} t_{0} v_{A}}=\Sigma_{\left\{x_{B}-x_{A}\right\} t_{0} v_{B}}=\Sigma_{v_{A} v_{B}}=[0]
$$

\section{Conclusion}

This article introduces rigorous matrix equations to transform coordinates of points and their velocities from one arbitrary terrestrial frame to another. The seven similarity transformation parameters required in Eqs. (12), (13), and (14) for transforming coordinates and/or velocities between different ITRF frames were given in Boucher and Altamimi (1996), with the restriction mentioned in Sect. 5. Parameters for the transformation between WGS84 (G873) and ITRF94 are presented in Malys et al. (1997b). The new ITRF96 is aligned to ITRF94 in origin, scale, orientation, and time evolution. Recently determined transformation parameters between the GLONASS-defined frame (P90, also referred to as PE-90) and the WGS84 were reported in the literature (Misra et al. 1996; Langley 1997). Similarly, the transformation of GPS processed vector components could be obtained using identical set of parameters and Eq. (25). The rigorous application of this methodology assumes the knowledge of the original velocities of the points involved. Presently, only points pertaining to the CORS and ITRF GPS networks have an associate velocity field. However, approximate estimates of absolute velocity components on a terrestrial frame could be determined using Eq. (15) and an adopted plate tectonic model such as NNR-NUVEL1A. Using the same or equivalent plate models, changes in the coordinates of any point due to plate rotations could be computed according to Eq. (18), where $R_{\ell}(\Omega)$ is given by Eq. (17) as a function of the direction cosines of the rotation axis or, alternatively, by Eq. (22) if the geographic location of the rotation axis pole is known. Finally, Eq. (23) expresses the more intuitive variation in curvilinear geodetic coordinates of a point as a function of the components of the angular rotation of the plate where it is located or, alternatively, the point velocity components, along local three-dimensional coordinate frames.

\section{Appendix}

The rigorous similarity transformation between two sets of coordinates $\{x\}_{A}$ and $\{x\}_{B}$ referred respectively to frames $A$ and $B$ expressed symbolically by the mapping $A \rightarrow B$ can be written:

$$
\{x\}_{B}=\left\{T_{x}\right\}+\sigma[\mathfrak{R}]\{x\}_{A}
$$

where $\left\{T_{x}\right\}$ was previously defined in Eq. (12), $\sigma$ is the scale factor, and $[\mathfrak{R}]$ is the orthogonal matrix rotating frame $A$ into $B$ (anticlockwise rotations are assumed positive).

The coordinates of any arbitrary point moving with velocity components $\{\dot{x}\}_{A\left(t_{0}\right)}$ referred to the old frame, will change during the interval $t_{0}$ to $t$ according to: 
$\{x\}_{A(t)}=\{x\}_{A\left(t_{0}\right)}+\{\dot{x}\}_{A\left(t_{0}\right)}\left(t-t_{0}\right)$

Similarly, the coordinates $\{x\}_{B(t)}$ of any point on frame $B$ at time $t$ which moved from $t_{0}$ to $t$ with velocity $\{\dot{x}\}_{B\left(t_{0}\right)}$ is determined by:

$\{x\}_{B(t)}=\{x\}_{B\left(t_{0}\right)}+\{\dot{x}\}_{B\left(t_{0}\right)}\left(t-t_{0}\right)$

Equation (A1) relating frames $A$ and $B$ can be written at any initial time $t_{0}$ :

$\{x\}_{B\left(t_{0}\right)}=\left\{T_{x}\right\}+\sigma[\mathfrak{R}]\{x\}_{A\left(t_{0}\right)}$

The variation of these coordinates with time is given by:

$$
\begin{aligned}
\{\dot{x}\}_{B\left(t_{0}\right)}= & \left\{\dot{T}_{x}\right\}+\llbracket \dot{\sigma}[\mathfrak{R}]+\sigma[\dot{\mathfrak{R}}] \rrbracket\{x\}_{A\left(t_{0}\right)} \\
& +\sigma[\mathfrak{R}]\{\dot{x}\}_{A\left(t_{0}\right)}
\end{aligned}
$$

Therefore, in order to know the transformed coordinates on frame $B$ at time $t$ the above two equations should be substituted into Eq. (A3), to obtain:

$$
\begin{aligned}
\{x\}_{B(t)}= & \left\{T_{x}\right\}+\sigma[\mathfrak{R}] \llbracket\{x\}_{A\left(t_{0}\right)}+\{\dot{x}\}_{A\left(t_{0}\right)}\left(t-t_{0}\right) \rrbracket \\
& +\llbracket\left\{\dot{T}_{x}\right\}+[\dot{\sigma}[\mathfrak{R}]+\sigma[\dot{\mathfrak{R}}]]\{x\}_{A\left(t_{0}\right)} \rrbracket\left(t-t_{0}\right)
\end{aligned}
$$

Under the assumption of differential rotations, the matrix $[\mathfrak{R}]$ reduces to $[\epsilon]^{t}+[I]$, where $[\epsilon]^{t}$ is given by Eq. (2). It is also common practice in the geodetic literature to express the final coordinates in terms of the corrections to the original coordinates. If this logic is applied to differential rotations and scale changes, one has:

$$
\{x\}_{B}=\{x\}_{A}+[\epsilon]^{t}\{x\}_{A}=\left[[\epsilon]^{t}+[I]\right]\{x\}_{A}
$$

and instead of writing $\{x\}_{B}=\sigma\{x\}_{A}$ as assumed previously, the changes to the original coordinates due to scale variations could be written:

$$
\{x\}_{B}=\{x\}_{A}+s\{x\}_{A}=(1+s)\{x\}_{A}
$$

When the simplifications $[\mathfrak{R}]=[\epsilon]^{t}+[I]$ and $\sigma=1+s$ are applied to Eq. (A1), the standard seven parameter similarity transformation expression is obtained (e.g., Lambeck 1971; Leick and Gelder 1975, p. 4):

$$
\{x\}_{B}=\left\{T_{x}\right\}+(1+s) \llbracket[\epsilon]^{t}+[I] \rrbracket\{x\}_{A}
$$

If the second-order differential products of the scale factor by the rotations are neglected, the preceding equation takes the alternative explicit form:

$$
\begin{aligned}
\left\{\begin{array}{l}
x \\
y \\
z
\end{array}\right\}_{B}= & \left\{\begin{array}{l}
x \\
y \\
z
\end{array}\right\}_{A}+\left\{\begin{array}{l}
T_{x} \\
T_{y} \\
T_{z}
\end{array}\right\} \\
& +\left[\begin{array}{ccc}
s & \epsilon_{z} & -\epsilon_{y} \\
-\epsilon_{z} & s & \epsilon_{x} \\
\epsilon_{y} & -\epsilon_{x} & s
\end{array}\right]\left\{\begin{array}{l}
x \\
y \\
z
\end{array}\right\}_{A}
\end{aligned}
$$

Equation (A10) is the form given by Boucher and Altamimi (1996). However, it should be stressed that these authors assumed clockwise rotations of coordinate axes to be positive.

If the simplifications for $[\Re]$ and $\sigma$ described above are introduced into Eq. (A6), after grouping common terms Eqs. (12) and (13) are obtained.

\section{References}

Argus DF, Gordon RG (1991) No-net-rotation model of current plate velocities incorporating plate rotation model NUVEL-1. Geophys Res Lett 18: 2039-2042

Bevis M, Taylor FW, Schutz BE, Recy J, Isacks BL, Helu S, Singh R, Kendrick E, Stowell J, Taylor B, Calmant S (1995) Geodetic observations of very rapid convergence and back-arc extension at the Tonga arc. Nature 374: 249-251

Borkowsky KM (1989) Accurate algorithms to transform geocentric to geodetic coordinates. Bull Geod 63: 50-56

Boucher C, Altamimi Z (1996) International terrestrial reference frame. GPS World 7: 71-74

DeMets C, Gordon RG, Argus DF, Stein S (1994) Effect of recent revisions to the geomagnetic reversal time scale on estimates of current plate motions. Geophys Res Lett 21: 2191-2194

Euler L (1775) Nova methodus motum corporum rigidorum determinandi. Novi Commentarii Academiae Scientiarum Imperialis Petropolitanae 20: 208-238. Reprinted in (1968). Opera Omnia (2) 9: 99-125, Societatis Scientiarum Naturalium Helveticae, Basileae

Feissel M, Gambis D (1993) The International Earth Rotation Service: current results for research on Earth rotation and reference frames. Adv Space Res 13(11): 143-150

GPS World (1997) Arbitrary alterations. GPS World 8(2):12

Heiskanen WA, Moritz H (1967) Physical geodesy. WH Freeman, San Francisco

Kaula WM (1966) Theory of satellite geodesy. Blaisdell, Waltham, Mass

Lambeck K (1971) The relation of some geodetic datums to a global geocentric reference system. Bull Geod 99: 37-53

Langley RB (1997) GLONASS: review and update. GPS World 8(7): 46-51

Leick A, Gelder BHW van (1975) On similarity transformations and geodetic network distortions based on Doppler satellite observations. Rep 235, Dept Geod Sci Surv, The Ohio State University, Columbus, Ohio

Malys S, Slater JA, Smith RW, Kunz LE, Kenyon SC (1997a) Status of the World Geodetic System 1984. In: Proc Int Symp Kinematic systems in geodesy, geomatics and navigation, Banff, Canada, 1977. Dept Geomatics Eng, Univ Calgary, pp 25-34

Malys S, Slater JA, Smith RW, Kunz LE, Kenyon SC (1997b) Refinements to the World Geodetic System 1984. In: Proc 10th Int Tech Meeting of the satellite division of The Institute of Navigation, Kansas City, MO, 1977. The Institute of Navigation, Alexandria VA, pp 841-850

McCarthy D (ed) (1996) IERS technical note 21. Observatoire de Paris, France

Misra PN, Abbot RI, Gaposchkin EM (1996) Transformation between WGS84 and P-90. In: Proc 9th Int Tech Meeting of the satellite division of The Institute of Navigation, Kansas City, MO, 1996. The Institute of Navigation, Alexandria VA, pp 307314

Mueller II (1969) Spherical and practical astronomy as applied to geodesy. Ungar, New York

Neilan RE (1997) IGS organization and the international tracking network. In: Zumberge JF, Fulton DE, Neilan RE (Eds) International GPS service for geodynamics 1996 Annual Report, Jet Propulsion Lab, Pasadena, Calif, pp 23-38

Snay RA, Cline MW, Philipp CR, Jackson DD, Feng Y, Shen ZK, Lisowski M (1996) Crustal velocity field near the big bend of California's San Andreas fault. J Geophys Res 101: 3173-3185 
Soler T (1976) On differential transformations between Cartesian and curvilinear (geodetic) coordinates. Rep 236. Dept Geod Sci Surv, Ohio State Univ, Columbus, Ohio

Soler T, Carlson AE Jr, Evans AG (1989) Determination of vertical deflections using the Global Positioning System and geodetic leveling. Geophys Res Lett 16: 695-698

Strange WE (1995) A national spatial system framework: continuously operating reference stations. In: IGS in government.
Proc First Federal Geographic Technology Conference. GIS World Books, Fort Collins, CO, pp 37-41

Thomson W (Lord Kelvin), Tait PG (1879) Principles of mechanics and dynamics (formerly titled Treatise on natural philosophy), Part one. Cambridge University Press, Cambridge. Reprinted (1944) Dover Publications, New York 